\title{
Fish Experimental Organism Diagnosis
}

National Cancer Institute

\section{Source}

National Cancer Institute. Fish Experimental Organism Diagnosis. NCI Thesaurus. Code C136559.

A condition that is relevant to either disease states or models of disease in fish. 